\title{
Wilson Prize article: Reflections on our experiences with developing the theory of intrabeam scattering"
}

\author{
Anton Piwinski, ${ }^{1, \dagger}$ James D. Bjorken, ${ }^{2, \$}$ and Sekazi K. Mtingwa, ${ }^{3, \S}$ \\ ${ }^{1}$ DESY, 22607 Hamburg, Germany \\ ${ }^{2}$ SLAC National Accelerator Laboratory, Menlo Park, California 94025, USA \\ ${ }^{3}$ Massachusetts Institute of Technology, Cambridge, Massachusetts 02139, USA
}

(Received 16 August 2018; published 7 November 2018)

\begin{abstract}
We discuss our experiences with developing detailed theoretical descriptions of intrabeam scattering in particle accelerators. We focus on the historical importance of understanding intrabeam scattering for the successful operation of a variety of accelerators around the world. In doing so, we highlight the fact that the theoretical understanding of intrabeam scattering played a crucial role in the discovery of the top quark at Fermilab, intermediate vector bosons $\mathrm{W}^{ \pm}, \mathrm{Z}$ and the Higgs particle at CERN, and the perfect liquid quarkgluon plasma at Brookhaven's Relativistic Heavy Ion Collider. We describe several useful high energy approximations to intrabeam scattering, including those that utilize a Modified Piwinski high energy approximation by Karl Bane that has gained wide usage in applications to electron damping rings and advanced light sources. Finally, we comment on the fact that a detailed understanding of intrabeam scattering at synchrotron-based advanced light sources is empowering many transformational discoveries in a myriad of disciplines.
\end{abstract}

DOI: $10.1103 /$ PhysRevAccelBeams.21.114801

\section{INTRODUCTION}

Intrabeam scattering (IBS) involves multiple small-angle Coulomb scatterings of charged particles within accelerator beams. This phenomenon leads to the growth in beam emittances, which places severe limitations on luminosity lifetimes in hadron and heavy ion colliders and the ability to achieve ultrasmall beam emittances in intense electron storage rings. The latter are used in damping rings for linear colliders, as well as synchrotron-based advanced light sources.

The detailed theory of IBS is described in a number of publications [1-6]. Reference [7] contains the most general treatment of IBS that includes both derivatives of accelerator lattice functions and linear coupling caused by skew quadrupoles and solenoids. Moreover, the result is represented as a single integral.

\footnotetext{
"The authors received the American Physical Society's 2017 Robert R. Wilson Prize for Outstanding Achievement in the Physics of Particle Accelerators. This paper is an invited contribution inspired by that award.

apiwinski@web.de

†bjbjorken@gmail.com

${ }^{\S}$ sekazi.mtingwa@gmail.com,

Published by the American Physical Society under the terms of the Creative Commons Attribution 4.0 International license. Further distribution of this work must maintain attribution to the author(s) and the published article's title, journal citation, and DOI.
}

When attempting to use the full theory in many software codes, it takes a long time to compute the dependence of longitudinal and transverse emittances on variables such as elapsed time and bunch charge. Thus, over the years, many authors have derived high energy approximations to the full theory that are more computationally friendly [8-13]. In particular, Bane [13] has described a modification of the Piwinski theory [1] at high energies and showed its equivalence at high energies to the theory described in Ref. [4].

This paper describes our roles in the development of a comprehensive theory of IBS, for which we received the American Physical Society's 2017 Robert R. Wilson Prize for Outstanding Achievement in the Physics of Particle Accelerators, with the citation

For the detailed, theoretical description of intrabeam scattering, which has empowered major discoveries in a broad range of disciplines by a wide variety of accelerators, including hadron colliders, damping rings/ linear colliders, and low emittance synchrotron light sources.

We are deeply honored to receive this prestigious prize. Our individual testimonies sometimes will be denoted by $\mathrm{Bj}$ (as he is known to his friends and colleagues) for James Bjorken, SM for Sekazi Mtingwa, and AP for Anton Piwinski.

In the next section, AP discusses a related phenomenon called the Touschek effect and his experiences with 
TABLE I. Coulomb scattering of particles within a beam.

Touschek effect

(1) Single scattering

(2) Considers only energy transfer from transverse to longitudinal oscillations.

(3) A small transverse momentum is transformed into a large change of longitudinal momentum (multiplied by the Lorentz factor $\gamma$ ).

(4) There is no change of particle distribution, but loss of both colliding particles.
Intrabeam scattering developing the first comprehensive theory of IBS that quantifies simultaneous changes of emittances in all three dimensions. In Secs. III and IV, respectively, $\mathrm{Bj}$ and SM share their experiences with using quantum field theory techniques to develop a theory of IBS that includes the case of strong-focusing accelerators, wherein the lattice parameters, which describe the bending and focusing effects of the magnets on the circulating beam, vary around the accelerator. In Sec. V, we summarize the full AP and Bj-SM formulas, introduce Bane's Modified Piwinski approximation, and present several high energy IBS approximations. In Sec. VI, we discuss the important role that IBS plays in the performance of synchrotron-based advanced light sources. Finally, in Sec. VII, we offer concluding comments.

\section{REFLECTIONS BY ANTON PIWINSKI ON THE TOUSCHEK EFFECT AND THE FIRST COMPREHENSIVE THEORY OF INTRABEAM SCATTERING}

I first heard about IBS in 1973 while attending a workshop in Italy. I recall learning that IBS could be a serious problem in proton accelerators. Since DESY was discussing at that time a new proton storage ring, I decided to investigate this problem.

In the literature, I found several reports on the Touschek effect, but little about IBS. The Touschek effect and IBS are two different aspects of the same phenomenon, namely Coulomb scattering within a charged particle beam. For the Touschek effect, only large scattering angles are taken into account. On the other hand, for IBS, small scattering angles are considered, and it is assumed that all changes of coordinates due to the scattering are small compared to the beam dimensions. This is summarized in Table I, which shows the main characteristics and differences between IBS and the Touschek effect.

For the Touschek effect, sufficient theoretical investigations existed that allowed for a reasonable calculation of the beam lifetime due to the effect. Figure 1 shows the first measurement of the Touschek effect in 1963 [14]. The inverse of the lifetime is plotted as a function of the number of electrons stored in the beam. As seen, the lifetime varies from 6 to 50 hours. The measurements were done in the small storage ring Anello di Accumulazione (AdA), which is the Italian word for storage ring, at an energy of $188 \mathrm{MeV}$. In the figure, there is a small displacement above zero, which is caused by the scattering of the electrons off the residual gas. In order to explain this measurement, Bruno Touschek found the relativistic mechanism for the longitudinal momentum change. To simplify the calculation, he made some approximations, e.g., he assumed nonrelativistic particle velocities in the center-of-mass system of the beam and he assumed a flat beam, i.e., no vertical betatron oscillations of the electrons.

Table II shows the successive improvements of the theory. After the first measurements and explanation by Touschek and others in 1963 [14], the lifetime was determined for ultrarelativistic energies, i.e., for relativistic energies in the center-of-mass system by Gittelmann and Ritson at SLAC [15]. In 1965, Völkel of DESY finally generalized the theory for arbitrary energies [16]. Then in 1998, I extended the theory to arbitrary beam sizes, removing the restriction to flat beams, thereby taking into account vertical betatron oscillations [17]. Moreover, I included nonzero derivatives of the accelerator lattice

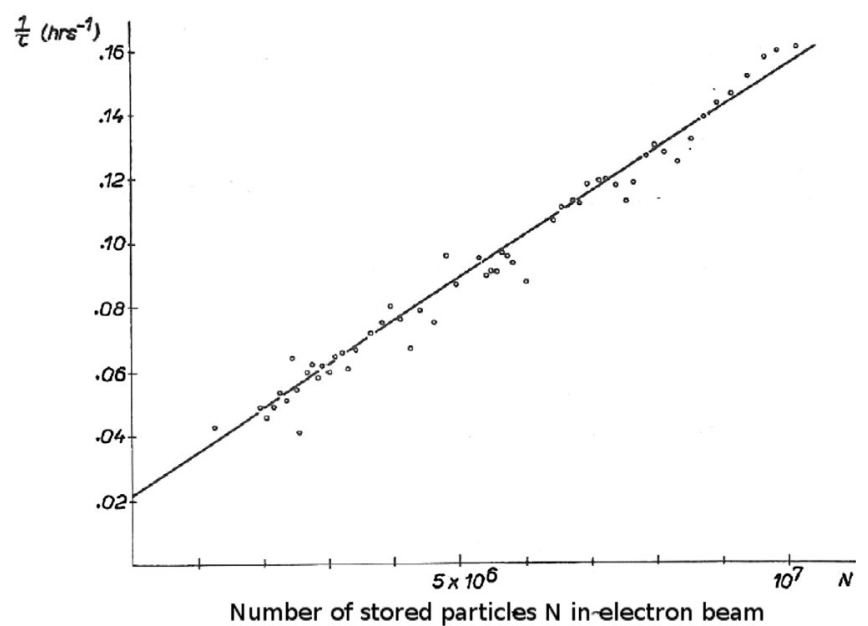

FIG. 1. First measurement of the Touschek effect in 1963 [14]. Inverse lifetime $\frac{1}{\tau}$ versus $N$, the number of stored particles in the beam, at energy $E=188 \mathrm{MeV}$. 
TABLE II. Historical notes for the Touschek effect.

\begin{tabular}{ll}
\hline \hline Reference & \multicolumn{1}{c}{ Comments } \\
\hline$[14]$ & $\begin{array}{l}\text { First measurement of this effect and explanation by B. Touschek assuming nonrelativistic } \\
\text { center-of-mass velocities and flat beam } \\
\text { Calculation of the rise time for ultrarelativistic energies }\end{array}$ \\
{$[15]$} & $\begin{array}{l}\text { Calculation for arbitrary energies } \\
{[16]}\end{array}$ \\
{$[17]$} & Consideration of the vertical betatron oscillations and a variation of the beam envelopes \\
\hline \hline
\end{tabular}

functions that describe the effect of the magnets that guide and focus the beam.

Turning next to IBS, there were only a few attempts to estimate the transfer of oscillation energy from one direction to another, mainly from transverse to longitudinal, i.e., from betatron oscillations to synchrotron oscillations, but not vice versa, which is necessary for a realistic description. Indeed, IBS is the mutual exchange of oscillation energies among all three directions and only by taking into account all energy transfers can one obtain a complete description.

Figure 2 shows a typical example of a measurement of this effect that was performed in 1984 at CERN's Super Proton Synchrotron (SPS) at an energy of $300 \mathrm{GeV}$. It depicts the longitudinal distribution of a proton bunch on the left side and an antiproton bunch on the right side at different times. The time difference between successive curves is a quarter of an hour. The main difference between the two sets of curves is the particle density, or number of particles in a bunch, with the density of the proton bunch being an order of magnitude larger than that of the antiproton bunch. Consequently, the growth of the proton bunch length is clearly stronger than that of the antiproton bunch. Similar measurements also were made for the bunch width and for the bunch height. All such measurements in

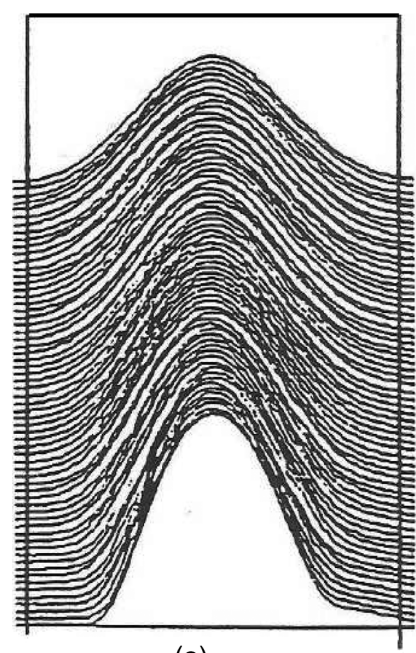

(a)

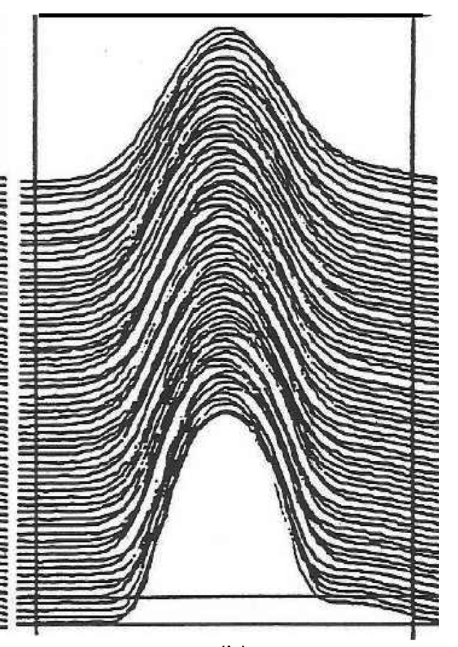

(b)
FIG. 2. Measurements made in the CERN SPS at energy $300 \mathrm{GeV}$ of the longitudinal distribution of (a) a proton bunch $\left(N^{+}=1.5 \times 10^{11}\right)$ and (b) an antiproton bunch $\left(N^{-}=1.2 \times 10^{10}\right)[3]$. various storage rings show such a slow change of the beam dimensions.

Table III shows a brief review of early IBS investigations. Bruck and Le Duff in 1964 [18] and Pellegrini in 1966 [19] performed the first investigations, where they calculated the transfer of oscillation energy from one direction to another. In 1974, I included, for the first time, energy exchanges among all three directions, including energy losses due to transfers from one direction to the other [1]. Thus at last, my work allowed one to calculate the development of the beam dimensions over a long time.

In 1977, Simon van der Meer was working on his new idea about the stochastic cooling of beams, for which he would later receive the Nobel Prize. He asked for the most precise calculation of the rise times due to IBS, because stochastic cooling would have to compete with IBS. Thus, Sacherer and I independently derived formulas that included derivatives of the lattice amplitude function $\beta$ and of the dispersion $\eta$. Hübner, Möhl, and Sacherer incorporated this work into a computer program at CERN, which researchers used at a number of accelerator facilities. Then in 1983, Bjorken and Mtingwa used quantum field theory to develop a completely new and elegant theory for IBS [4].

The rise times $\tau_{s, x, z}$ for the three dimensions can be written in the following form:

$$
\frac{1}{\tau_{s, x, z}}=d \cdot F\left(a_{s, x, z}, b_{s, x, z}\right) / \gamma^{4} .
$$

Here $d$ is the particle density in phase space, namely

$$
d=\frac{N}{\sigma_{s} \sigma_{p} \sigma_{x} \sigma_{x^{\prime}} \sigma_{z} \sigma_{z^{\prime}}}
$$

with $\mathrm{N}$ being the number of particles, $\gamma$ is the Lorentz factor, and the scattering function $F$ considers the imbalance of the oscillation energies for the three directions, or in other words, the imbalance of the temperatures for the three directions since it depends only on $a_{s, x, z}$ and $b_{s, x, z}$, which are the ratios of bunch dimensions. Here, I do not show the explicit form of the scattering function $F$, which will be discussed in more detail later in a slightly different form as defined in Ref. [1]. It is complicated and contains an integral which usually must be solved numerically. It can be positive, negative, or zero. However, it can be zero for all 
TABLE III. Historical notes for intrabeam scattering.

\begin{tabular}{ll}
\hline \hline Reference & \multicolumn{1}{c}{ Comments } \\
\hline$[18,19]$ & $\begin{array}{l}\text { Investigation of the transfer of oscillation energy from one direction to another } \\
\text { Investigation of energy transfer among all three directions taking into account the } \\
\text { corresponding energy losses } \\
\text { Calculation of rise times due to IBS including the derivatives of lattice amplitude and } \\
\text { dispersion functions (A. Piwinski, F. Sacherer) } \\
\text { A completely new and elegant method for the calculation of the rise times including } \\
\text { the derivatives of the lattice functions }\end{array}$ \\
{$[4]$} & \\
\hline \hline
\end{tabular}

three directions at the same time only at energies below transition energy. Above transition energy, at least one of the three rise times must be different from zero.

This behavior can be seen with help of the following invariant that can be derived from the three rise times:

$$
\left(\frac{1}{\gamma^{2}}-\alpha_{M}\right)\left\langle\frac{\Delta^{2} p}{p^{2}}\right\rangle_{\mathrm{avg}}+\left\langle x^{\prime 2}\right\rangle_{\mathrm{avg}}+\left\langle z^{\prime 2}\right\rangle_{\mathrm{avg}}=\mathrm{constant},
$$

where

$p, \Delta p=$ momentum and momentum spread

$x^{\prime}, z^{\prime}=$ horizontal and vertical betatron angles.

The momentum compaction factor $\alpha_{M}$ is the ratio of the relative orbit lengthening to the corresponding relative energy change. The average has to be taken over all particles and the whole circumference. Below transition energy $\left(\gamma_{t r}=1 / \sqrt{\alpha_{M}}\right)$, the first bracket in Eq. (3) is positive and all oscillation amplitudes are limited. Here an equilibrium distribution can exist. Above transition energy, the first bracket is negative and all oscillation amplitudes can grow as far as they do not exceed other limitations, e.g., the chamber wall.

This invariant is correct only if the derivatives of $\beta$ and dispersion $\eta$ are neglected. If they are taken into account, the above mentioned expression is not constant but increases slowly. The derivatives appear always in the combination $\eta^{\prime} \beta-\beta^{\prime} \eta / 2$. Therefore, the contributions from large $\beta^{\prime}$ in the interaction regions ( $>100$ ) vanishes since the dispersion in interaction regions is usually zero. The invariant can be considered as an approximation that shows in principle the different behaviors of IBS below and above transition energy.

\section{REFLECTIONS BY JAMES BJORKEN ON A QUANTUM FIELD THEORY APPROACH TO INTRABEAM SCATTERING}

It is a great pleasure and privilege to be a recipient of the 2017 Robert R. Wilson Prize. I am sure that there are a lot of people unaware that I ever had anything to do with the theory of particle accelerators. But for a long time, I have been very proud to have earned a membership card in the union of accelerator theorists. However, I never dreamed that it would come to this.

My interest in the subject began in the 1970s at SLAC, thanks in large part to my close association and friendship with Burton Richter. He put into my hands the classic Matt Sands tutorial on electron storage rings [22]. When I moved to Fermilab in 1979, I vowed to learn about proton machines as well. By 1981, I had progressed enough that, as I recall, I was something of a groupie within the community of Fermilab accelerator theorists. Then, in the summer of 1981, Alvin Tollestrup introduced me to the IBS problem, which he had been working on himself. As already discussed by Anton, there had been a lot of prior work, the most important being by Anton himself. But the most general case of a strong-focusing machine lattice was not yet fully understood. And at Fermilab, this case needed to be understood in the context of the design of the Antiproton Accumulator ring and of what is now known as the Tevatron.

My recollection of the details, not to mention my comprehension of the subject matter, has greatly deteriorated in the more than three decades since that time. But I am a packrat, and found a fat file full of notes from that period. From them, it appears that I rather quickly got up to speed on the problem. In retrospect, the reason for this lay in my experiences in the world of particle-physics theory. A bunch of 10 billion protons traveling down a beampipe at nearly the speed of light is not totally dissimilar from an ion containing a hundred nucleons doing the same thing, or even a single relativistic nucleon containing all those quarks and gluons, also doing the same thing. So it appears to me in hindsight that I was, from the start, in something of a comfort zone, and could apply the manifestly-relativistically-invariant formalisms developed for particle theory, especially by Feynman, to this problem.

Evidence for this exists in my own handwritten notes, dated August 1981, which are in particle physics language, and which exhibit for sure a fresh approach to the problem. Evidently, the first problem facing me was whether I could reproduce what Piwinski had already done. On page 8 of my first note appears the sentence, "Translate into ordinary lingo". By page 9, I had moved into the accelerator physics language: there is a line, "We follow Piwinski in defining 
the following variable..." And by page 11, the conclusion was, "This agrees with Piwinski's formula, although it may still be accidental". Two days after this first note, there was created a second one, which rephrased and streamlined the computations present in the first one. The key mathematical tactic was a famous identity used by Feynman and Schwinger to evaluate integrals associated with Feynman diagrams. It is not clear to me whether there was a genuine "aha" moment in that two-day interim.

And this is about the time that Sekazi Mtingwa, who had just completed a postdoctoral position in the Fermilab theoretical physics group, and was transitioning to a new position, came to me looking for a problem. IBS was, as I recall, all I could offer to him. But Sekazi, despite having to start from scratch in learning the trade, signed on. So it is possible that the reason I wrote those two notes was to provide him with something better than the chaotic scribbling, barely intelligible to me, that I used when working alone.

Between the fall of 1981 and the spring of 1982, the documentation in my file is rather sparse. What is clear in retrospect is that during that period, Sekazi was rapidly riding up the learning curve, and more and more of the problem landed in his capable hands. There was all through that period a close working relationship with Alessandro Ruggiero, the resident Fermilab accelerator theorist most deeply involved in the IBS problem. He produced several internal notes during that period, and is acknowledged in our paper as well. And in my file is a short message from Anton, indicating that he was during that period also up to speed. We found ourselves in agreement on the results, although there was a pesky overall factor of two that had to be negotiated amongst us.

By the summer of 1982, Sekazi and I had created a draft of our paper. All the equations therein are in Sekazi's handwriting. I am quite sure that by that time he had essentially taken over from me, especially with regard to crunching the numbers. It turned out that for the Fermilab designs, IBS effects did not need to be viewed with much alarm. But as you will hear from Sekazi, this has not always been the case.

In a nutshell, the physics idea expressed in our paper is that, viewed in the rest frame of the bunch, IBS tends to make the bunch grow in size, and to evolve toward isotropy in momentum space. On the other hand, accelerator designers impose strong, time-dependent electromagnetic fields that squeeze and stretch the bunch in ways designed to inhibit such behavior. The formulas that we derived exhibit this physics somewhat more transparently than what had been done before.

The above story is an example of the importance of cross-fertilization. In those days it was especially easy for me to cross over from particle physics to accelerator physics. I did not have to go through an annual performance review, demonstrating how my activities were contributing to the goals of the elementary particle physics theory group, as defined by some set of oversight committees. Nowadays it is harder to engage in crossover research or in research topics outside of the mainstream.

I am at present interested in the dark energy problem, which places me again as an amateur, this time within the world of general relativity. And I see again opportunities for crossover activity. One has to do with gravitational lensing. The standard methodology uses the language of ray-tracing optics. I do not see any significant use of the Hamiltonian language of Courant, Livingston, and Snyder, an approach which has revolutionized the field of particlebeam optics for more than a half century. I cannot help but wonder whether this approach could be productive when applied to gravitation.

Another such area is in the formalism of general relativity itself. The predominant choice of language is that of the century-old Einstein-Hilbert description, where the basic degrees of freedom are the 10 independent components of the metric tensor. Almost as old is the description advocated by Cartan, often called gauge gravity, where the degrees of freedom are the 24 components of an $\mathrm{O}(3,1)$ gauge potential called the spin connection, supplemented by 16 more called the vierbein, out of which the standard metric tensor is constructed. The EinsteinCartan language is distinctly different from the EinsteinHilbert language, although the physics consequences are nearly, but not totally, identical. In particular, if one wants to incorporate Dirac particles as gravitational sources, one is obliged to utilize the Einstein-Cartan language. For sure, this gauge-gravity language is familiar to essentially all general relativity theorists. But, as I see it, it is in the bones of only a minority of them. I wish that there were more of a balance.

The distinction between Einstein-Hilbert and EinsteinCartan gravity is a more grandiose version of what occurs in Higgs physics. The language of the nonlinear sigma model, with its three degrees of freedom, is enough to describe the Higgs mechanism, which gives the electroweak gauge bosons their mass. But one needs the linear sigma model, with its four degrees of freedom, to account for the famous Higgs particle itself.

In conclusion, I want to add my congratulations and best wishes to the co-recipients of this prize, Anton Piwinski and Sekazi Mtingwa, along with heartfelt thanks to Herman Winick for his enthusiastic support.

\section{REFLECTIONS BY SEKAZI MTINGWA ON HIS FORAY INTO INTRABEAM SCATTERING AND ACCELERATOR PHYSICS}

Just as $\mathrm{Bj}$, I also would like to credit the excellent Matt Sands tutorial [22] on electron storage rings for providing me with my introduction to the basic theory of particle accelerators. After wrestling with those concepts and Anton's beautiful and highly condensed paper on IBS 
[1], I began to see how Bj's and my field of quantum field theory could add to the discourse and even make a number of IBS phenomena more transparent. As already mentioned by $\mathrm{Bj}$, we finished a draft of our paper during the summer of 1982 and submitted it to the journal, Particle Accelerators, where it was published.

It was about this time that I actually made a foray into experimental work. Fred Mills, who was in charge of magnet design and construction at the Antiproton Source, asked me to help him to develop an analytic approach for designing the endpacks to be installed on either end of each magnet so that the integrated field through each magnet would meet the design specifications. We succeeded in this important task. Each magnet that was fabricated would have its integrated field measured and we would calculate how to design the corrective endpacks. Fortunately, we were spot on for each magnet, greatly reducing the time and expense required to produce the Antiproton Source dipoles and quadrupoles. It turns out that our colleagues at Michigan State University were constructing an accelerator during the early 1980s and Fred shared our approach with them to speed their process along.

With two accelerator victories under my belt, I formally joined the Antiproton Source Stochastic Beam Cooling Group in 1983. Since Bj and I had just published our IBS paper, stochastic cooling was a natural fit for me to further my rapidly growing interest in accelerator physics. There I worked closely with John Marriner in finalizing the vacuum and beam sensitivity designs of the pickup and kicker electrodes. Glen Lambertson and his colleagues performed much of the early work at Lawrence Berkeley National Laboratory (LBNL), where they designed and constructed the prototype devices. I was detailed to Fermilab's technical staff that fabricated the pickups and kickers, where I performed quality assurance tests to ensure their microwave performance, collaborated with James Simpson and colleagues at Argonne National Laboratory's $20 \mathrm{MeV}$ electron linac in performing beam tests on LBNL prototype electrodes, and oversaw the installation of the pickups and kickers into the Debuncher and Accumulator Accelerators in the Antiproton Source tunnel. I even had the good fortune to be featured in the August 1985 issue of Ebony Magazine, where there appeared a photo of me standing next to one of the large stochastic cooling tanks.

As depicted in Fig. 3, in the beginning years, the Antiproton Source consisted of a target station, beam transport lines, and two small accelerators called the Debuncher and Accumulator, which were both contained in the same tunnel. Protons were extracted from the Main Ring at $120 \mathrm{GeV}$ and impinged upon a tungsten-rhenium target, whereby a Li lens would focus secondary particles

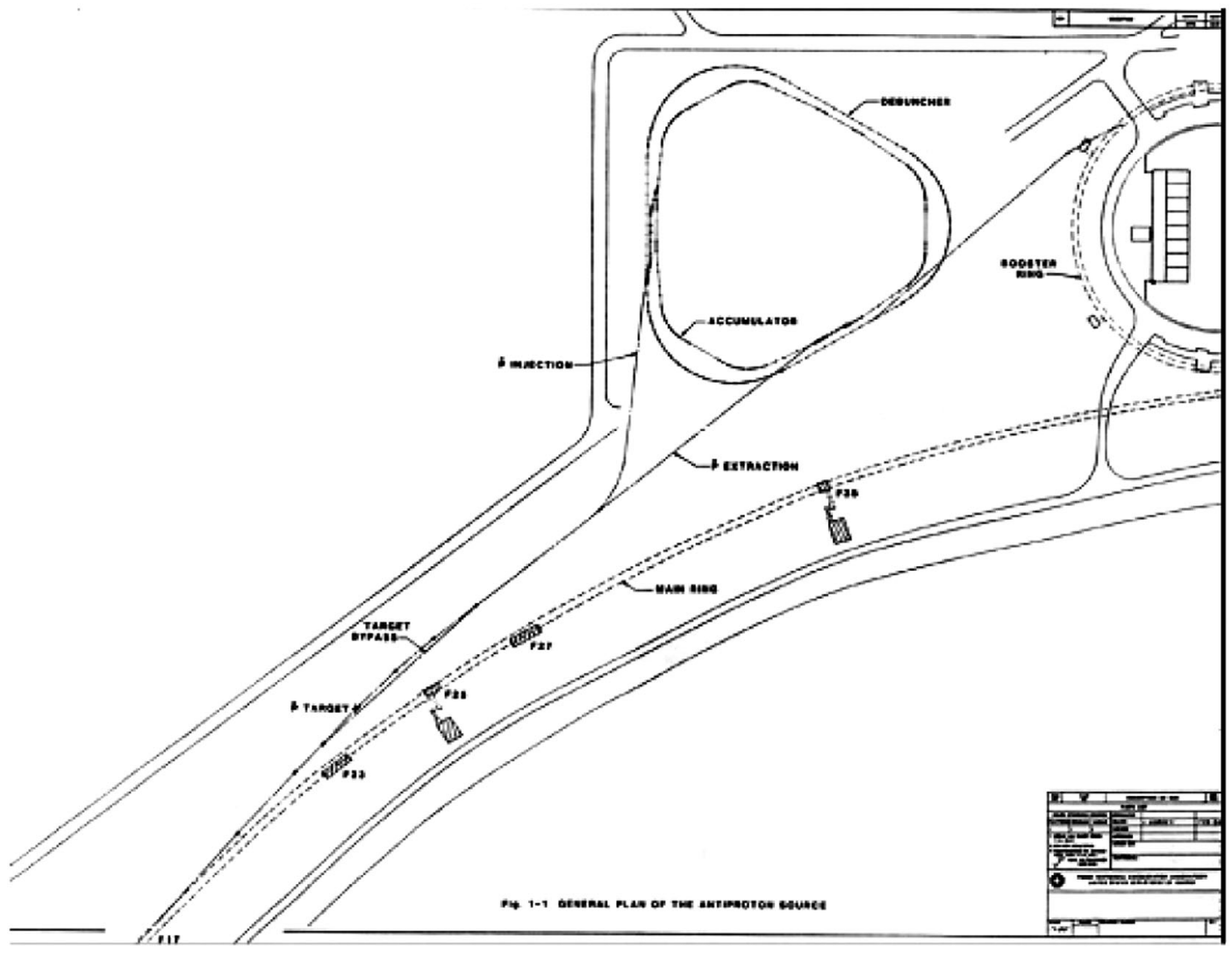

FIG. 3. Antiproton Source (From Fermilab). 
off the target, and a pulsed dipole magnet would steer 8.9 GeV antiprotons toward the Debuncher. This accelerator converted the antiproton bunches into a continuous beam and began the process of cooling it, namely reducing its momentum spread and transverse phase space. The final cooling and accumulation of the antiprotons into a high-density core in momentum space occurred in the Accumulator. At the time, there was considerable anxiety that IBS would hamper the efforts to achieve the goal of stacking $4 \times 10^{11}$ antiprotons in the core every 4 hours. That was the genesis of Bj's and my interest in gaining a better understanding of IBS for strong-focussing accelerators with their varying lattice parameters. In the end, our theoretical analyses and numerical simulations showed that IBS would not be a problem, so we were all greatly relieved.

A particle accelerator is one of the most complex scientific devices to design, construct, and operate. There are many systems that must work in tandem and to high precision. At the Tevatron complex, I was involved in the construction of the two systems already mentioned: Antiproton Source magnet and stochastic cooling. Relative to the latter, there were 23 large tanks, 12 in the Debuncher and 11 in the Accumulator, each containing numerous delicate, even brittle, pickup and kicker electrodes. John Marriner and I were responsible for ensuring that those intricate devices worked once commissioning commenced. The Antiproton Source worked well over the following decade, being a crucial element in the 1995 discovery of the top quark. I strongly feel that I and all my accelerator colleagues at the Antiproton Source and Tevatron should be counted as codiscoverers of the top quark, given the extreme sweat and tears that it took to put those accelerator systems into place for the detectors. I think that our high energy physics community should take a serious look at devising a system to reward those on the accelerator end with coauthorship of papers involving major discoveries.

Since those early years of the Antiproton Source, many improvements and upgrades were made, including to the stochastic cooling systems. Around the year 2000, a decade after Bj and I had left Fermilab, IBS finally caught up with the laboratory. The journal, Science, featured a story on the lab's problems [23]. I quote:

A year and a half ago, the Tevatron, which smashes protons and antiprotons together at enormous energies, began operating again after a $\$ 260$ million refit. Despite months of tinkering, however, scientists and engineers couldn't boost the beam's luminosity-its brightness-high enough to begin the bulk of the accelerator's research program...

A major problem with the accelerator lies in the system that accumulates, accelerates, and stores antiprotonswhich, unlike protons, are hard to produce. Fully $80 \%$ of the antiprotons were supposed to survive the trip from the accumulator system to the collider, but in January, a mere 30\% made the journey intact. "Really, until April we had no idea what the physical cause of this problem was", says [Stephen] Holmes [Head of Fermilab's Beams Division]. So, despite Fermilab's best efforts, "we topped out at about 40\%. We were pretty much stuck".

In April, however, scientists at Fermilab figured out that the antiproton problem was caused by intrabeam scattering. "When the antiprotons are going around and around in the antiproton accumulator, they are confined to a very small space, and they are bouncing off each other", says Holmes. "This tends to heat the beam, making it get bigger. It wants to blow up". Scientists had anticipated problems, but this effect was worse than expected.

"Now a 2-week shutdown in June might have solved the antiproton problem", Holmes says. While the accelerator was turned off, engineers improved the beam cooling system and refocused the magnetic optics that keeps the beam tight. Now about 50\% to 60\% of the antiprotons survive the trip to the accelerator, and the number is rising. With that roadblock removed, last week the Tevatron's luminosity surged to a recordsetting $2.64 \times 10^{31}$ inverse square centimeters per second...

Not long after $\mathrm{Bj}$ and I completed our work, Alvin Tollestrup, who led Fermilab's experimental team that eventually discovered the top quark, informed me that he was looking for ways to simplify Piwinski's scattering function so that he could use it for studying upgrades to the Tevatron lattice. Alvin is one of those rare physicists who can design both detectors and accelerator lattices. As he tinkered with lattice designs for the first Tevatron upgrade, he wanted to know the effects of IBS on luminosity lifetime for any changes he made to the lattice, without having to wait long periods of time running computer programs. He asked if I would be interested in collaborating with him to obtain simple analytic expressions for the Piwinski scattering function found in Eq. (1), since that would greatly reduce the computer time required. Given the approximately $1 \mathrm{TeV}$ energy of the protons and antiprotons at the Tevatron, we had the advantage of using approximations to the Piwinski theory for asymptotically large energies.

Alvin and I succeeded in completely integrating the Piwinski scattering function at high energies [9], and arrived at a simplified new scattering function that is useful for predicting the evolution of luminosity with time for the Tevatron and future generations of hadron colliders. This will be discussed in the next section. Our result allowed Alvin to proceed with his work on the accelerator lattice for the first Tevatron upgrade. Moreover, one of our Fermilab colleagues, David Finley, used our results and my paper with $\mathrm{Bj}$ to study the effects of IBS on the proposed Tevatron upgrade's integrated luminosity and demonstrated that, 
while IBS effects were visible, they did not negate gains made by adjusting other accelerator parameters [24].

Approximately 15 years passed before I engaged with IBS again. I became interested in the next generation electron-positron collider and joined the team that led to the International Linear Collider (ILC) collaboration. My Lawrence Berkeley National Laboratory colleagues, William Barletta, Miguel Furman and Andy Wolski invited me to spend some time with Andy working on IBS for the ILC damping rings. KEK had already begun studies in their prototype damping ring called the Accelerator Test Facility (ATF). Karl Bane at SLAC had spent some time there and had proposed an elegant modification of the Piwinski theory that connects Piwinski's formulas to Bj's and my formulas at high energies [13], which was quite applicable to the $1.28 \mathrm{GeV}$ electron beams at the ATF damping ring. Some time ago, Martini incorporated varying lattice parameters into the Piwinski theory [5]. Over the years, others have done so for certain high energy approximations, such as discussed in Refs. [8,10-12]. However, by using Bane's modification of Piwinski's formulas, one is able to introduce the derivatives of the accelerator lattice functions into the Piwinski theory in a natural way and show the connection to the work by $\mathrm{Bj}$ and me.

Kiyoshi Kubo, one of the lead researchers at the ATF, Andy and I succeeded in combining Alvin's and my work with Bane's Modified Piwinski approximation to arrive at what we called a Completely Integrated Modified Piwinski (CIMP) solution to IBS [25]. We then used it to obtain excellent numerical analyses for the ATF data and also used it in the design of the ILC damping rings. Later, I was able to derive completely integrated formulas for the IBS growth times for flat beams [26], which are typical for electron/positron damping rings and synchrotron light sources, wherein the vertical emittance is much smaller than the horizontal emittance. These high energy approximations will be discussed in the next section.

\section{INTRABEAM SCATTERING FORMULAS, BANE'S MODIFICATION OF PIWINSKI'S FORMULAS, AND HIGH ENERGY APPROXIMATIONS}

In this section, we review the main IBS formulas, introduce Bane's Modified Piwinski approximation, and present the high energy approximations mentioned in the previous section. For this discussion, we change the notation for $x, z$ used in Sec. II to $h, v$ for horizontal and vertical directions.

\section{A. Piwinski's formulas}

We start by stating AP's main results. According to Ref. [1] and summarized nicely in Ref. [2], the rise times are given by

$$
\begin{aligned}
& \frac{1}{\tau_{p}}=A\left\langle\frac{\sigma_{h}^{2}}{\sigma_{p}^{2}} f(\tilde{a}, \tilde{b}, \tilde{q})\right\rangle \\
& \frac{1}{\tau_{h}}=A\left\langle f\left(\frac{1}{\tilde{a}}, \frac{\tilde{b}}{\tilde{a}}, \frac{\tilde{q}}{\tilde{a}}\right)+\frac{\eta_{h}^{2} \sigma_{h}^{2}}{\beta_{h} \varepsilon_{h}} f(\tilde{a}, \tilde{b}, \tilde{q})\right\rangle \\
& \frac{1}{\tau_{v}}=A\left\langle f\left(\frac{1}{\tilde{b}}, \frac{\tilde{a}}{\tilde{b}}, \frac{\tilde{q}}{\tilde{b}}\right)+\frac{\eta_{v}^{2} \sigma_{h}^{2}}{\beta_{v} \varepsilon_{v}} f(\tilde{a}, \tilde{b}, \tilde{q})\right\rangle,
\end{aligned}
$$

with

$$
A=\frac{r_{0}^{2} c N}{64 \pi^{2} \beta^{3} \gamma^{4} \varepsilon_{h} \varepsilon_{v} \sigma_{s} \sigma_{p}},
$$

$N$ is the number of particles in a bunch, $r_{0}$ is the classical radius of the charged particle, $c$ is the speed of light in vacuum, $\beta$ is the particle speed divided by $c, \gamma$ is the particle energy divided by the rest mass, $\varepsilon_{h, v} \equiv \sigma_{h, v}^{2} / \beta_{h, v}$ are the transverse emittances, $\sigma_{h, v}$ are the rms transverse beam sizes, $\sigma_{s}$ is the rms bunch length, and $\sigma_{p}$ is the relative momentum spread. Also, $\beta_{h, v}$ and $\eta_{h, v}$ are the betatron and dispersion accelerator lattice functions, respectively, and $\langle\cdots\rangle$ indicates that the expression is to be averaged around the accelerator lattice. Also, we have

$$
\begin{aligned}
\frac{1}{\sigma_{h}^{2}} & =\frac{1}{\sigma_{p}^{2}}+\frac{\eta_{h}^{2}}{\beta_{h} \varepsilon_{h}}+\frac{\eta_{v}^{2}}{\beta_{v} \varepsilon_{v}} \\
\tilde{a} & =\frac{\sigma_{h}}{\gamma} \sqrt{\frac{\beta_{h}}{\varepsilon_{h}}} \\
\tilde{b} & =\frac{\sigma_{h}}{\gamma} \sqrt{\frac{\beta_{v}}{\varepsilon_{v}}} \\
\tilde{q} & =\sigma_{h} \beta \sqrt{\frac{2 d}{r_{0}}} .
\end{aligned}
$$

The maximum impact parameter $d$ [different from the $d$ in Eq. (1)] is usually taken to be the vertical beam size and the Piwinski scattering function $f$, which here is defined slightly differently from $F$ contained in Eq. (1) above, is defined in Ref. [1] as

$$
f(\tilde{a}, \tilde{b}, \tilde{q})=2 \int_{0}^{\infty} \int_{0}^{\pi} \int_{0}^{2 \pi} e^{-r\left[\cos ^{2} \theta+\left(\tilde{a}^{2} \cos ^{2} \phi+\tilde{b}^{2} \sin ^{2} \phi\right) \sin ^{2} \theta\right]} \ln \left(\tilde{q}^{2} r\right)\left(1-3 \cos ^{2} \theta\right) \sin \theta d \phi d \theta d r,
$$


where $f$ satisfies the following relations:

$$
\begin{gathered}
f(\tilde{a}, \tilde{b}, \tilde{q})=f(\tilde{b}, \tilde{a}, \tilde{q}) \\
f(\tilde{a}, \tilde{b}, \tilde{q})+\frac{1}{\tilde{a}^{2}} f\left(\frac{1}{\tilde{a}}, \frac{\tilde{b}}{\tilde{a}}, \frac{\tilde{q}}{\tilde{a}}\right)+\frac{1}{\tilde{b}^{2}} f\left(\frac{1}{\tilde{b}}, \frac{\tilde{a}}{\tilde{b}}, \frac{\tilde{q}}{\tilde{b}}\right)=0 .
\end{gathered}
$$

Evans and Zotter [3] performed two of the integrals in AP's scattering function giving

$$
\begin{aligned}
f(\tilde{a}, \tilde{b}, \tilde{q})= & 8 \pi \int_{0}^{1} d u \frac{\left(1-3 u^{2}\right)}{P Q} \\
& \times\left\{2 \ln \left[\frac{\tilde{q}}{2}\left(\frac{1}{P}+\frac{1}{Q}\right)\right]-0.577 \ldots\right\}
\end{aligned}
$$

with

$$
\begin{aligned}
& P^{2}=\tilde{a}^{2}+\left(1-\tilde{a}^{2}\right) u^{2} \\
& Q^{2}=\tilde{b}^{2}+\left(1-\tilde{b}^{2}\right) u^{2} .
\end{aligned}
$$

\section{B. Mtingwa-Tollestrup's high energy approximation to the Piwinski scattering function}

To obtain a completely integrated high energy approximation for the growth times in Eqs. (4)-(6), we follow the procedure in Ref. [9] and consider

$$
\frac{1}{\tau_{p}}=A\left\langle\frac{\sigma_{h}^{2}}{\sigma_{p}^{2}} f(\tilde{a}, \tilde{b}, \tilde{q})\right\rangle .
$$

For large $\gamma, \tilde{q}$ is much larger than $\tilde{a}$ and $\tilde{b}$. Thus, speaking in relative terms for the size of the arguments of $f$, we have $f$ (small, small, large). It is shown in Ref. [9] that whenever we have $f$ (large, small, large), then the integrals in $f$ can be done. Therefore, following Ref. [9], to convert $f$ (small, small, large) to an expression involving only $f$ 's of the form $f$ (large, small, large), we use the second Piwinski relation contained in Eq. (14). We arrive at

$$
f(\tilde{a}, \tilde{b}, \tilde{q})=-\frac{1}{\tilde{a}^{2}} f\left(\frac{1}{\tilde{a}}, \frac{\tilde{b}}{\tilde{a}}, \frac{\tilde{q}}{\tilde{a}}\right)-\frac{1}{\tilde{b}^{2}} f\left(\frac{1}{\tilde{b}}, \frac{\tilde{a}}{\tilde{b}}, \frac{\tilde{q}}{\tilde{b}}\right) .
$$

Thus, we now have

$$
\begin{aligned}
f(\text { small, small, large })= & -\frac{1}{\tilde{a}^{2}} f(\text { large, small, large }) \\
& -\frac{1}{\tilde{b}^{2}} f(\text { large, small, large }) .
\end{aligned}
$$

In such a case, where the first and third arguments of $f$ are large compared to the second, Ref. [9] gives in the high energy limit

$$
f(\alpha, \omega, \delta) \approx \frac{-4 \pi^{\frac{3}{2}} \ln \delta}{\alpha} g(\omega)
$$

where the Piwinski scattering function $f$ has been reduced to the function $g$, which is given in Ref. [9] by

$$
g(\omega)=\sqrt{\frac{\pi}{\omega}}\left[P_{-\frac{1}{2}}^{0}\left(\frac{\omega^{2}+1}{2 \omega}\right) \pm \frac{3}{2} P_{-\frac{1}{2}}^{-1}\left(\frac{\omega^{2}+1}{2 \omega}\right)\right],
$$

where $P_{\nu}^{-\mu}$ are the associated Legendre functions. One takes the plus sign for $\omega \geq 1$ and the minus sign for $\omega \leq 1$. Note that $g(\omega) \rightarrow \sqrt{\pi}$ as $\omega \rightarrow 1$ from above or below. We have found that the Type 3 associated Legendre functions are the correct ones to use. In any event, it is important to check that one can reproduce Table I in Ref. [9] for the function $g$.

\section{Bjorken-Mtingwa's formulas}

Next, we summarize the main formulas from Bj's and SM's work. The inverse rise times are

$$
\begin{gathered}
\frac{1}{\tau_{p}} \equiv \frac{1}{\sigma_{p}} \frac{d \sigma_{p}}{d t} \\
\frac{1}{\tau_{h}} \equiv \frac{1}{\varepsilon_{h}^{\frac{1}{2}}} \frac{d \varepsilon_{h}^{\frac{1}{2}}}{d t} \\
\frac{1}{\tau_{v}} \equiv \frac{1}{\varepsilon_{v}^{\frac{1}{2}}} \frac{d \varepsilon_{v}^{\frac{1}{2}}}{d t},
\end{gathered}
$$

with

$$
\begin{aligned}
\frac{1}{\tau_{i}}= & 4 \pi A(\log )\left\langle\int_{0}^{\infty} d \lambda \frac{\lambda^{\frac{1}{2}}}{[\operatorname{det}(L+\lambda I)]^{\frac{1}{2}}}\right. \\
& \left.\times\left\{\operatorname{Tr} L^{i} \operatorname{Tr}\left(\frac{1}{L+\lambda I}\right)-3 \operatorname{Tr}\left[L^{i}\left(\frac{1}{L+\lambda I}\right)\right]\right\}\right\rangle,
\end{aligned}
$$

where $i$ represents $p, h$, or $v$. $A$ is defined in Eq. (7), and

$$
L=L^{(p)}+L^{(h)}+L^{(v)}
$$

$$
\begin{aligned}
L^{(p)} & =\frac{\gamma^{2}}{\sigma_{p}^{2}}\left(\begin{array}{lll}
0 & 0 & 0 \\
0 & 1 & 0 \\
0 & 0 & 0
\end{array}\right) \\
L^{(h)} & =\frac{\beta_{h}}{\varepsilon_{h}}\left(\begin{array}{ccc}
1 & -\gamma \phi_{h} & 0 \\
-\gamma \phi_{h} & \frac{\gamma^{2} \mathcal{H}_{h}}{\beta_{h}} & 0 \\
0 & 0 & 0
\end{array}\right)
\end{aligned}
$$

$$
L^{(v)}=\frac{\beta_{v}}{\varepsilon_{v}}\left(\begin{array}{ccc}
0 & 0 & 0 \\
0 & \frac{\gamma^{2} \mathcal{H}_{v}}{\beta_{v}} & -\gamma \phi_{v} \\
0 & -\gamma \phi_{v} & 1
\end{array}\right) .
$$


$(\log )$ is the Coulomb logarithm which we define later. Also, we have the horizontal dispersion invariant $\mathcal{H}_{h}=$ $\left[\eta_{h}^{2}+\left(\beta_{h} \eta_{h}^{\prime}-\frac{1}{2} \beta_{h}^{\prime} \eta_{h}\right)^{2}\right] / \beta_{h}$ and the function $\phi_{h}=\eta_{h}^{\prime}-$ $\frac{1}{2} \beta_{h}^{\prime} \eta_{h} / \beta_{h}$, with similar expressions for the vertical functions.

\section{Bane's modified Piwinski approximation}

To account for the lattice parameter variations around the accelerator in the high energy limit, Bane proposed [13] the following replacements in AP's formulas:

$$
\frac{\eta_{h}^{2}}{\beta_{h}} \rightarrow \mathcal{H}_{h}=\left[\eta_{h}^{2}+\left(\beta_{h} \eta_{h}^{\prime}-\frac{1}{2} \beta_{h}^{\prime} \eta_{h}\right)^{2}\right] / \beta_{h}
$$

which means that we can replace the parameters $\sigma_{h}, \tilde{a}, \tilde{b}, \tilde{q}$ with $\sigma_{H}, a, b, q$, namely

$$
\begin{aligned}
& \frac{1}{\sigma_{h}^{2}}=\frac{1}{\sigma_{p}^{2}}+\frac{\eta_{h}^{2}}{\beta_{h} \varepsilon_{h}}+\frac{\eta_{v}^{2}}{\beta_{v} \varepsilon_{v}} \rightarrow \frac{1}{\sigma_{H}^{2}}=\frac{1}{\sigma_{p}^{2}}+\frac{\mathcal{H}_{h}}{\varepsilon_{h}}+\frac{\mathcal{H}_{v}}{\varepsilon_{v}} \\
& \tilde{a}=\frac{\sigma_{h}}{\gamma} \sqrt{\frac{\beta_{h}}{\varepsilon_{h}}} \rightarrow a=\frac{\sigma_{H}}{\gamma} \sqrt{\frac{\beta_{h}}{\varepsilon_{h}}} \\
& \tilde{b}=\frac{\sigma_{h}}{\gamma} \sqrt{\frac{\beta_{v}}{\varepsilon_{v}}} \rightarrow b=\frac{\sigma_{H}}{\gamma} \sqrt{\frac{\beta_{v}}{\varepsilon_{v}}} \\
& \tilde{q}=\sigma_{h} \beta \sqrt{\frac{2 d}{r_{0}}} \rightarrow q=\sigma_{H} \beta \sqrt{\frac{2 d}{r_{0}}} .
\end{aligned}
$$

In the high energy limit, Bane showed that this modified Piwinski approximation gives excellent agreement with the work of Bj-SM.

\section{E. Kubo-Mtingwa-Wolski high energy completely integrated modified Piwinski (CIMP) approximation}

We now give the emittance growth times from Ref. [25] for the high energy CIMP approximation by invoking both Bane's modification of Piwinski's formulas and the scattering function of Tollestrup and SM, yielding

$$
\begin{aligned}
& \frac{1}{\tau_{p}} \approx A 2 \pi^{\frac{3}{2}}\left\langle\frac{\sigma_{H}^{2}}{\sigma_{p}^{2}}\left(\frac{\ln \left(\frac{q^{2}}{a^{2}}\right) g\left(\frac{b}{a}\right)}{a}+\frac{\ln \left(\frac{q^{2}}{b^{2}}\right) g\left(\frac{a}{b}\right)}{b}\right)\right\rangle \\
& \frac{1}{\tau_{h}} \approx A 2 \pi^{\frac{3}{2}}\left\langle-a \ln \left(\frac{q^{2}}{a^{2}}\right) g\left(\frac{b}{a}\right)\right. \\
& \left.+\frac{\mathcal{H}_{h} \sigma_{H}^{2}}{\varepsilon_{h}}\left(\frac{\ln \left(\frac{q^{2}}{a^{2}}\right) g\left(\frac{b}{a}\right)}{a}+\frac{\ln \left(\frac{q^{2}}{b^{2}}\right) g\left(\frac{a}{b}\right)}{b}\right)\right\rangle
\end{aligned}
$$

$$
\begin{aligned}
\frac{1}{\tau_{v}} \approx & A 2 \pi^{\frac{3}{2}}\left\langle-b \ln \left(\frac{q^{2}}{b^{2}}\right) g\left(\frac{a}{b}\right)\right. \\
& \left.+\frac{\mathcal{H}_{v} \sigma_{H}^{2}}{\varepsilon_{v}}\left(\frac{\ln \left(\frac{q^{2}}{a^{2}}\right) g\left(\frac{b}{a}\right)}{a}+\frac{\ln \left(\frac{q^{2}}{b^{2}}\right) g\left(\frac{a}{b}\right)}{b}\right)\right\rangle
\end{aligned}
$$

with $\sigma_{H}, a, b, q$, and $g$ defined in Eqs. (30)-(33), and (21).

We now turn our attention to the Coulomb log factor. Since $q$ is much larger than $a$ and $b$, in the CIMP approximation, we have $\ln \left(\frac{q^{2}}{a^{2}}\right) \approx \ln \left(\frac{q^{2}}{b^{2}}\right)$, so we can pull the common factor of $\ln \left(\frac{q^{2}}{a^{2}}\right) \equiv(\log )$ outside all the expressions. Finally, we obtain

$\frac{1}{\tau_{p}} \approx 2 \pi^{\frac{3}{2}} A(\log )\left\langle\frac{\sigma_{H}^{2}}{\sigma_{p}^{2}}\left(\frac{g\left(\frac{b}{a}\right)}{a}+\frac{g\left(\frac{a}{b}\right)}{b}\right)\right\rangle$

$\frac{1}{\tau_{h}} \approx 2 \pi^{\frac{3}{2}} A(\log )\left\langle-a g\left(\frac{b}{a}\right)+\frac{\mathcal{H}_{h} \sigma_{H}^{2}}{\varepsilon_{h}}\left(\frac{g\left(\frac{b}{a}\right)}{a}+\frac{g\left(\frac{a}{b}\right)}{b}\right)\right\rangle$

$\frac{1}{\tau_{v}} \approx 2 \pi^{\frac{3}{2}} A(\log )\left\langle-b g\left(\frac{a}{b}\right)+\frac{\mathcal{H}_{v} \sigma_{H}^{2}}{\varepsilon_{v}}\left(\frac{g\left(\frac{b}{a}\right)}{a}+\frac{g\left(\frac{a}{b}\right)}{b}\right)\right\rangle$,

where the common factor $(\log )$ that appears in Eqs. (25) and (37)-(39) is often taken to be

$$
(\log ) \equiv \ln \left(\frac{q^{2}}{a^{2}}\right) \approx \ln \left[\frac{\gamma^{2} \sigma_{v} \varepsilon_{h}}{r_{0} \beta_{h}}\right] .
$$

\section{F. Mtingwa's high energy approximation for flat beams}

To arrive at high energy IBS formulas for flat beams from Ref. [26], where the vertical emittance is much less than the horizontal, namely

$$
a^{2} \ll b^{2} \ll 1,
$$

where $a$ and $b$ are defined in Eqs. (31) and (32), an excellent approximation is achieved by dropping all offdiagonal elements in all matrices in Eqs. (27)-(29). This is typical of low-emittance electron and positron damping rings and synchrotron light sources. In fact, for the ATF at KEK, we average the parameters around the accelerator ring and obtain

$$
\begin{aligned}
& \left\langle a^{2}\right\rangle=0.00011 \\
& \left\langle b^{2}\right\rangle=0.03192 .
\end{aligned}
$$

We call dropping all off-diagonal elements in all matrices the diagonal matrices (DM) approximation. 
As discussed in Ref. [4], one can write the IBS emittance growth rates in terms of a diffusion matrix $K_{i j}$ as follows:

$$
\frac{1}{\tau_{a}}=\sum_{i j} K_{i j} L_{j i}^{(a)}
$$

where the matrices $L^{(a)}$ are defined in Eqs. (27)-(29) and

$$
\begin{aligned}
K_{i j}= & 4 \pi A(\log )\left\langle\int_{0}^{\infty} d \lambda \frac{\lambda^{\frac{1}{2}}}{[\operatorname{det}(L+\lambda I)]^{\frac{1}{2}}}\right. \\
& \left.\times\left\{\delta_{i j} \operatorname{Tr}\left(\frac{1}{L+\lambda I}\right)-3\left(\frac{1}{L+\lambda I}\right)_{i j}\right\}\right\rangle .
\end{aligned}
$$

In Ref. [26], these matrix elements are reduced to

$K_{i j}=0 \quad$ for $i \neq j$

$K_{11}=4 \pi A(\log ) \frac{\sigma_{H}^{2}}{\gamma^{2}}\left[\frac{6}{b}-2 b-\frac{4}{b} \tilde{K}\left(1-\frac{a^{2}}{2 b^{2}}\right)\right]$

$K_{22}=4 \pi A(\log ) \frac{\sigma_{H}^{2}}{\gamma^{2}}\left[-6+4 b+\frac{2}{b} \tilde{K}\left(1-\frac{a^{2}}{2 b^{2}}\right)\right]$

$K_{33}=4 \pi A(\log ) \frac{\sigma_{H}^{2}}{\gamma^{2}}\left[6-2 b-\frac{6}{b}+\frac{2}{b} \tilde{K}\left(1-\frac{a^{2}}{2 b^{2}}\right)\right]$,

where $\tilde{K}$ is the complete elliptic integral defined by

$$
\tilde{K}(k) \equiv F\left(\frac{\pi}{2}, k\right)
$$

with $F(\varphi, k)$ being the elliptic integral of the first kind and given by

$$
F(\varphi, k)=\int_{0}^{\varphi} \frac{d \alpha}{\sqrt{1-k^{2} \sin ^{2} \alpha}} .
$$

In Eqs. (47)-(49), we can use Hastings' approximation for $\tilde{K}(k)[27]$ :

$$
\tilde{K}(k)=\sum_{n=0}^{4} a_{n} t^{n}-\ln t \sum_{n=0}^{4} b_{n} t^{n}+\varepsilon(k),
$$

where $t=1-k^{2},|\varepsilon(k)| \leq 2 \times 10^{-8}$, and

$$
\begin{array}{ll}
a_{0}=1.38629436112 & b_{0}=0.5 \\
a_{1}=0.09666344259 & b_{1}=0.12498593597 \\
a_{2}=0.03590092383 & b_{2}=0.06880248576 \\
a_{3}=0.03742563713 & b_{3}=0.03328355346 \\
a_{4}=0.01451196212 & b_{4}=0.00441787012 .
\end{array}
$$

All of the above completely integrated high energy approximations for beam emittance growth rates greatly reduce the time it takes to compute average growth rates around accelerator lattices.

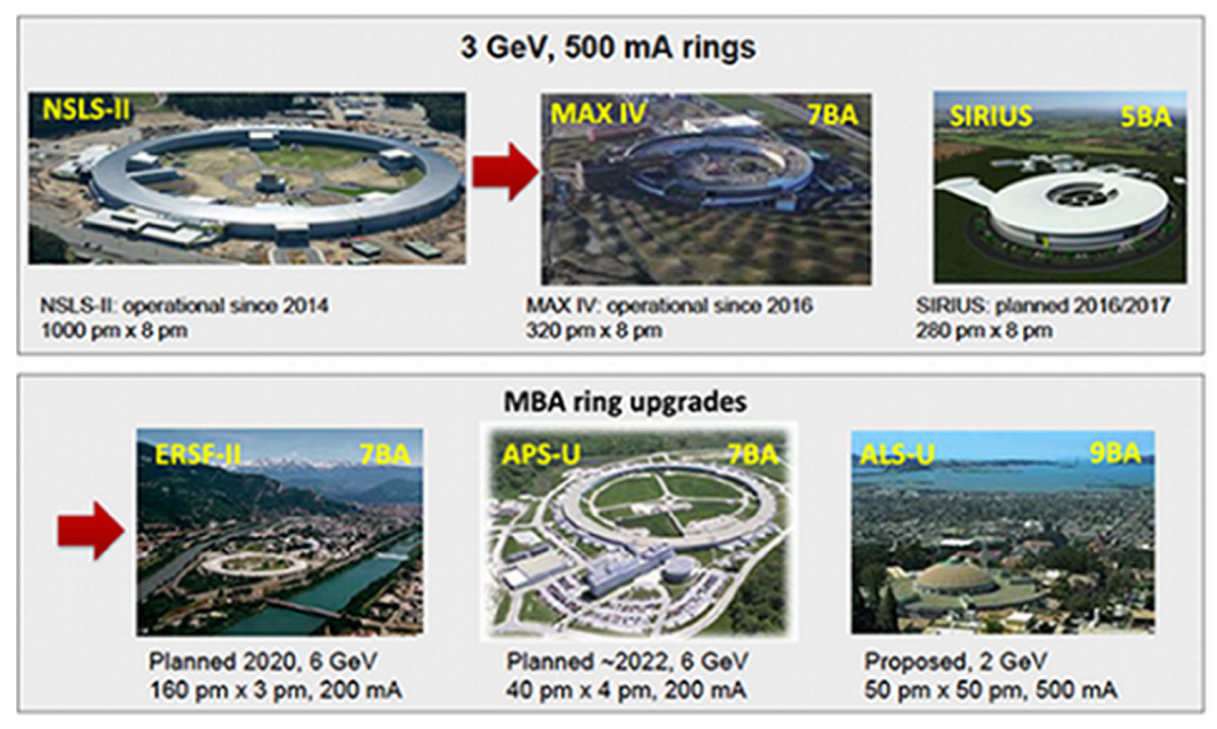

Other international implementations: Japan (SPring8-2, $6 \mathrm{GeV}$ ), China (HEPS, 5.6 GeV), Germany (PETRA-IV), France (SOLEIL), Switzerlond (SLS, 2.4 GeV), Itoly (ELETTRA) and others are developing plans

FIG. 4. Several post NSLS-II world advanced light sources implementing the new multibend achromat technology [28] (From MAX IV Laboratory). 


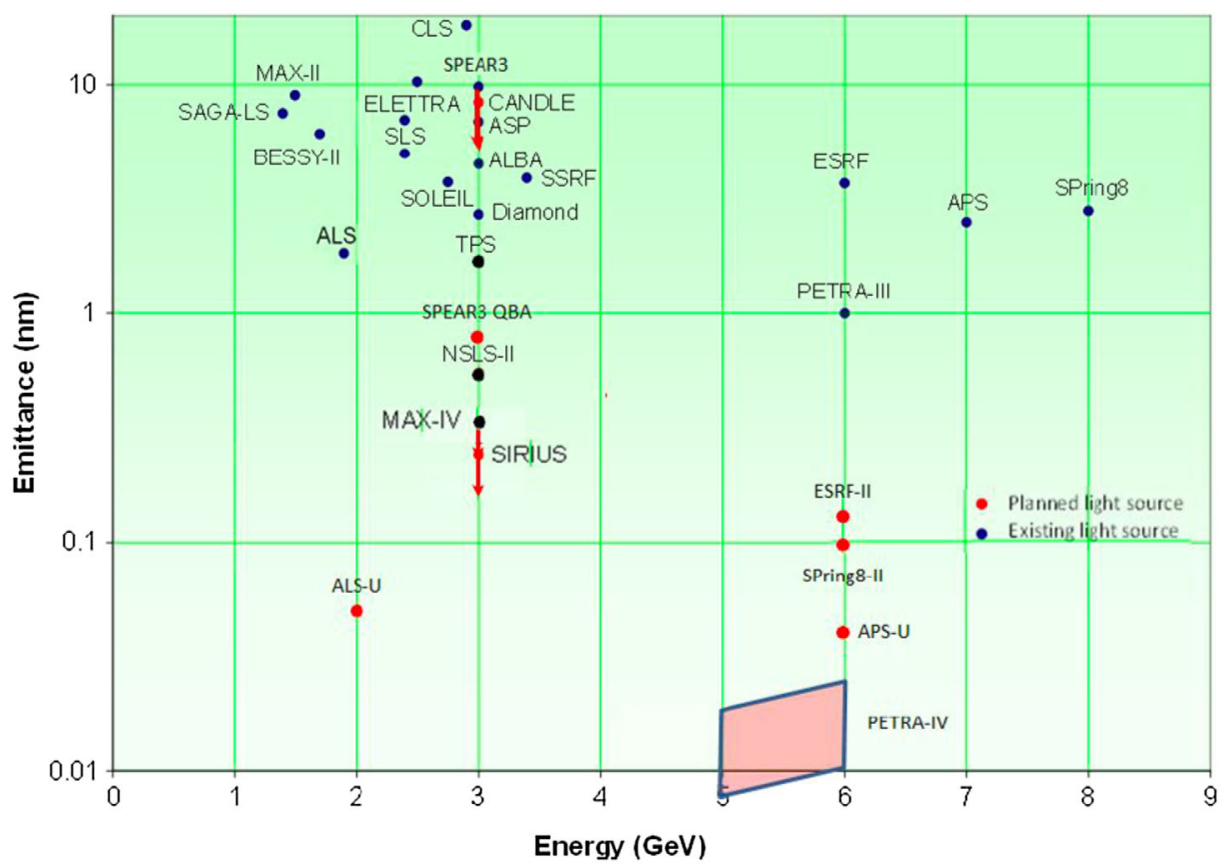

FIG. 5. Horizontal beam emittances at existing and planned world advanced light sources [28] Red = planned Blue = existing (From Argonne National Laboratory, managed and operated by UChicago Argonne, LLC, for the US Department of Energy under Contract No. DE-AC02-06CH11357).

\section{IBS AND THE MODERN GENERATION OF SYNCHROTRON LIGHT SOURCES}

Increasingly, IBS effects will be a crucial determining factor on the ultimate brightness of the latest designs of synchrotron-based advanced light sources. The new 4th generation advanced light sources make use of a new magnet design called the multibend achromat (MBA), which was invented by researchers in Sweden and implemented at their MAX IV light source. That and several other light sources implementing this new technology are shown in Fig. 4. Vertical electron beam emittances, which are the product of vertical beam size and vertical

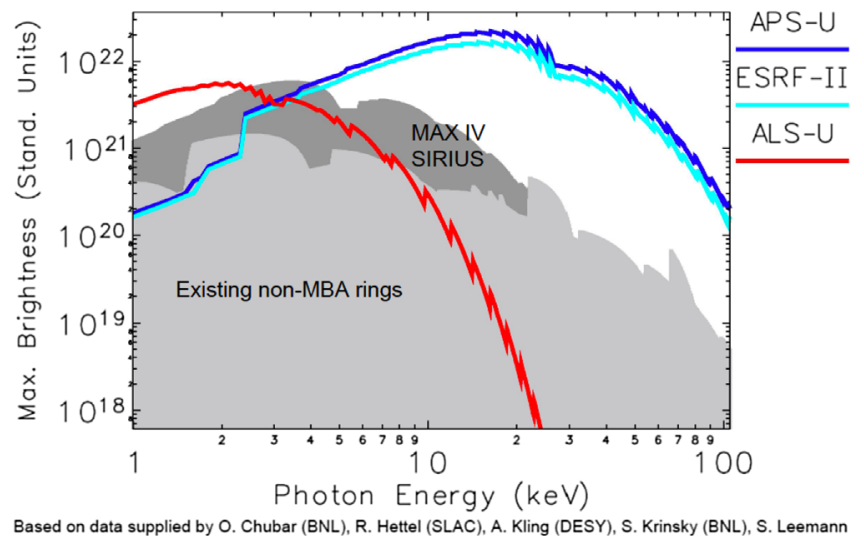
Based on data supplied by O. Chubar (BNL), R. Hettel (SLAC), A. Kling (DESY), S. Krinsky (BNL),
(MAX IV), T. Rabedeau (SLAC), P. Raimondi (ESRF), C. Steier (ALS), T. Watanabe (SPRing-8)

FIG. 6. Partial snapshot of the advanced light source brightness landscape [28] (From MAX IV Laboratory). divergence, are naturally quite small. In comparison, horizontal emittances tend to be many times larger. The smaller the horizontal electron emittance, the brighter photon beams tend to be that they generate. See the horizontal beam emittances in Fig. 5 and the resultant photon brightnesses in Fig. 6. With horizontal and vertical emittances of $\epsilon_{x}=320$ picometers and $\epsilon_{y}=8 \mathrm{pm}$, respectively, electron beam energy $E=3 \mathrm{GeV}$, current $I=500 \mathrm{~mA}$, and momentum acceptance $\delta_{p}=4.5 \%$,

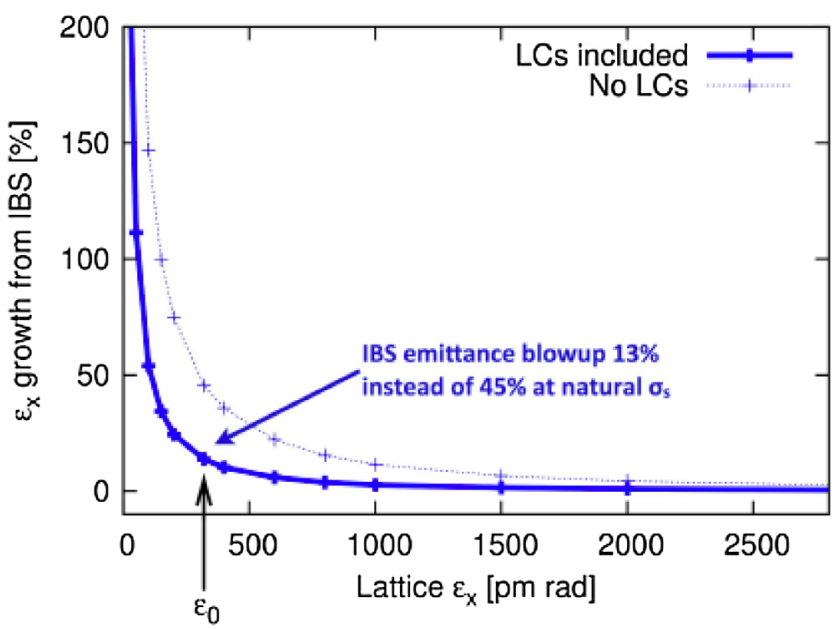

FIG. 7. \% Horizontal beam emittance growth due to intrabeam scattering $\mathrm{LCs}=$ Landau cavities, $\left(\varepsilon_{x} ; \varepsilon_{y}\right)=(320 \mathrm{pm} ; 8 \mathrm{pm})$ $E=3 \mathrm{GeV} ; I=500 \mathrm{~mA} ; \delta_{p}=4.5 \%$ [28] (From MAX IV Laboratory). 


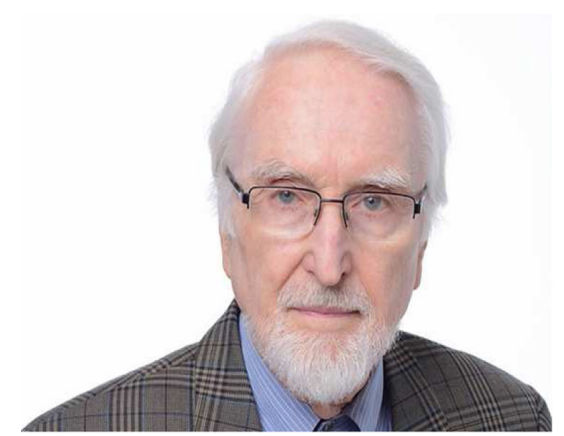

(a)

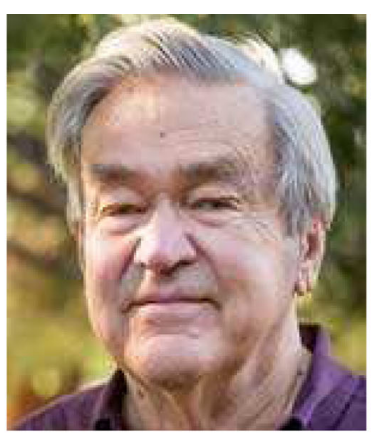

(b)

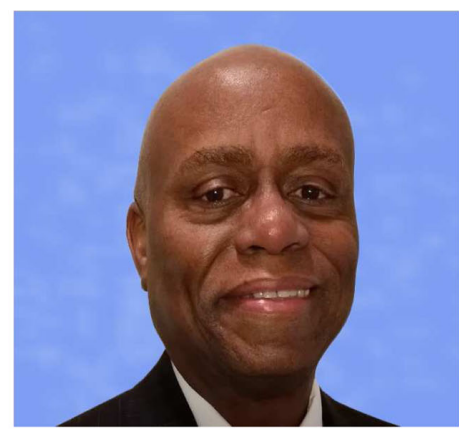

(c)

FIG. 8. Photos of (a) Anton Piwinski, (b) James Bjorken, and (c) Sekazi Mtingwa.

Fig. 7 [28] shows the Max IV \% horizontal beam emittance growth with and without devices called Landau cavities (LCs), which help to mitigate the adverse effects of IBS.

Figure 7 shows that, more and more, IBS will be a stringent limitation that must be overcome in future light sources. It is a dominant heating mechanism for all high intensity beams, constraining luminosity lifetimes in hadron colliders and determining equilibrium emittances in antiproton accumulators, electron and positron damping rings, and advanced light sources.

\section{CONCLUSION}

As accelerator and high energy physicists, we are proud that our community invented the synchrotrons used for advanced light sources, which are arguably the most transformative scientific instruments since the invention of conventional lasers and computers. Advanced light sources are revolutionizing a myriad of fundamental and applied sciences, including agriculture, biology, biomedicine, chemistry, climate and environmental sciences, cultural heritage studies, energy, engineering, geology, materials science, nanotechnology, paleontology, pharmaceutical discoveries, and physics, with an accompanying impact on industrial and economic development. As a community, we need to add advanced light sources to our list of technological breakthroughs that have changed the way we live, along with the World Wide Web and various medical imaging techniques.

In conclusion, AP's [Fig. 8(a)] interest in IBS is traced to his interest in understanding the operation of DESY's proposed proton storage ring in the early 1970s. When $\mathrm{Bj}$ [Fig. 8(b)] and SM [Fig. 8(c)] undertook their work, they were trying to understand its effects on Fermilab's Antiproton Source's ability to accumulate high quality antiproton beams and on the Tevatron's luminosity lifetime. Little did any of us know that, over several decades, understanding and quantifying the effects of IBS would become so crucial for the successful operation of such a wide class of accelerators, even playing important roles in the discoveries of the long sought top quark at Fermilab, intermediate vector bosons $\mathrm{W}^{ \pm}, \mathrm{Z}$ and the Higgs particle at CERN, the perfect liquid quark-gluon plasma at Brookhaven's Relativistic Heavy Ion Collider, and helping to revolutionize so many disciplines at advanced light sources.

We would like to extend our heartfelt thanks to Herman Winick, Alvin Tollestrup, and Leon Lederman for their enthusiastic support and others who supported us both during the time of our work and for receipt of the 2017 Wilson Prize.

[1] A. Piwinski, in Proceedings of the 9th International Conference on High Energy Accelerators, Stanford, CA, USA, 1974, p. 405.

[2] A. Piwinski, in Handbook of Accelerator Physics and Engineering, edited by A. Chao and M. Tigner (World Scientific, Singapore, 1999), p. 125.

[3] L. Evans and B. Zotter, Report No. CERN-SPS-80-15, 1980.

[4] J. Bjorken and S. Mtingwa, Part. Accel. 13, 115 (1983).

[5] M. Martini, Report No. CERN-PS-AA-84-9, 1984.

[6] S. Nagaitsev, Intrabeam scattering formulas for fast numerical evaluation, Phys. Rev. ST Accel. Beams 8, 064403 (2005).

[7] A. Piwinski, Report No. DESY 90-113, 1990.

[8] G. Parzen, Intrabeam scattering at high energies, Nucl. Instrum. Methods Phys. Res., Sect. A 256, 231 (1987).

[9] S. Mtingwa and A. Tollestrup, Report No. Fermilab-Pub89/224, 1987.

[10] J. Le Duff, in Proceedings of the CERN Accelerator School, Berlin, Germany, 1987 (CERN, Geneva, 1989), p. 114.

[11] T. Raubenheimer, Ph.D. Thesis, Stanford University, Report No. SLAC-R-387, 1991, Sec. II.3.1.

[12] J. Wei, in Proceedings of the Particle Accelerator Conference, Washington, DC, USA, 1993 (IEEE, Piscataway, 1993), p. 3651.

[13] K. Bane, in Proceedings of the 8th European Particle Accelerator Conference, Paris, 2002 (EPS-IGA and CERN, Geneva, 2002), p. 1443.

[14] C. Bernardini, G. F. Corazza, G. Di Giugno, G. Ghigo, J. Haissinski, P. Marin, R. Querzoli, and B. Touschek, Lifetime and Beam Size in a Storage Ring, Phys. Rev. Lett. 10, 407 (1963).

[15] B. Gittelmann and D. M. Ritson, Stanford University Report No. HELP-291, 1963. 
[16] U. Völkel, Report No. DESY 67/5, 1965.

[17] A. Piwinski, Report No. DESY 98-179, 1998.

[18] H. Bruck and J. Le Duff, Beam enlargement in storage rings by multiple Coulomb scattering, Technical Report, Laboratoire de l'Accélérateur Linéaire, Orsay, France (1964).

[19] C. Pellegrini, in Proc. Int. Symposium on Elec. Pos. Storage Rings (1966).

[20] K. Hübner, D. Möhl, and F. Sacherer, CERN computer code, 1977.

[21] R. Giannini and D. Möhl, CERN Report No. PS/AR/Note 92-22, 1993.

[22] M. Sands, SLAC Report No. 121, 1970.

[23] C. Seife, Tevatron sees light at end of tunnel?, Science 297, 757 (2002).
[24] D. Finley, Report No. Fermilab-TM-1646, 1989.

[25] K. Kubo, S. Mtingwa, and A. Wolski, Intrabeam scattering formulas for high energy beams, Phys. Rev. ST Accel. Beams 8, 081001 (2005).

[26] S. Mtingwa, A new high energy approximation of intrabeam scattering for flat electron and positron beams, Afr. Phys. Rev., 2, 0001 (2008).

[27] For an excellent discussion, see S. Zhang and J. Jin, Computation of Special Functions (John Wiley \& Sons, New York, 1996), p. 661.

[28] M. Borland, R. Hettel, S. C. Leemann, and D. S. Robin, from Accelerator Physics Challenges in the Design of Multi-Bend-Achromat-Based Storage Rings, Presentation at the North American Particle Accelerator Conference (NAPAC 2016), Chicago, IL, USA. 